\title{
Evaluación de la densidad mineral ósea en niños con infección vertical por VIH
}

\author{
Claudia Torrejón', M. Isabel Galaz², Eloísa Vizueta, Ana M. Álvarez', Elba Wü4, Ana Chávez5, Julia Villarroel6, \\ Karla Yohannessen", Mariana Hevia7, Maritza Vivanco ${ }^{8}$, Constanza Rivero y Paulina Balboa ${ }^{7}$
}

'Departamento de Pediatría y Cirugía Infantil campus Norte Universidad de Chile. ¿Unidad de Infectología, Hospital Roberto del Río.

${ }^{3}$ Unidad de Infectología Hospital San Borja Arriarán.

${ }^{4}$ Unidad de de Infectología

Hospital San Juan de Dios. 5Unidad de Infectología Hospita

Exequiel González Cortés ${ }^{6}$ Unidad de Infectología Hospita Félix Bulnes Cerda.

Departamento de Pediatría

Universidad de los Andes.

¿Unidad de Endocrinología, Hospital Roberto del Río.

${ }^{9}$ Escuela de Nutrición, Universidad de Chile.

Los autores declaran no tener potencial conflicto de intereses alguno.

Fuente de financiamiento: código del proyecto FAI MED-002-12.

Recibido: 1 de septiembre de 2017

Aceptado: 25 de octubre de 2018

Correspondencia a: Claudia Torrejón Silva: torrejón_clau@yahoo.com

\section{Introducción}

L a terapia anti-retroviral de gran actividad (TARGA) mejora la supervivencia y el crecimiento de los niños con infección por el virus de inmunodeficiencia humana (VIH). Sin embargo, su uso puede estar asociado a cambios adversos en la composición corporal, el metabolismo y la mineralización ósea ${ }^{1}$.

En adultos se ha observado que la disminución de la densidad mineral ósea (DMO) es más común en los pacientes con infección por VIH que en la población general, lo que hace que este grupo sea más susceptible a fracturas ${ }^{2}$. Paccout y cols. ${ }^{3}$, en un meta-análisis, encontraron una prevalencia de $15 \%$ de osteoporosis y $52 \%$ de osteopenia en adultos infectados por el VIH. En los niños con con esta infección, existen pocos estudios que evalúen la DMO y no hay datos chilenos ${ }^{1,4-6}$.

Los mecanismos sugeridos para el desarrollo del compromiso óseo son la existencia de una inflamación crónica, viremia incontrolada, la promoción de la apoptosis osteoblástica y la proliferación de los osteoclastos producidos por la enfermedad y anti-retrovirales (ARV) usados ${ }^{7}$, la deficiencia de vitamina $\mathrm{D}$ (principalmente en pacientes con infección perinatal por $\mathrm{VIH})^{2}$, toxicidad mitocondrial ${ }^{8}$, pérdida de peso, retraso del crecimiento 9 y pubertad tardía ${ }^{10}$.
Durante la infancia y la adolescencia, la masa ósea aumenta junto al crecimiento longitudinal y los cambios en el tamaño y la forma del esqueleto. A los 18 años de edad, se alcanza al menos $90 \%$ de la masa ósea máxima y al final de la tercera década de vida, la adquisición es completa ${ }^{11}$. Teniendo en cuenta que la masa ósea es un importante predictor de fracturas y osteoporosis en la vida adulta, es muy importante lograr una masa ósea adecuada antes de llegar a la edad adulta.

\section{Objetivo}

Evaluar la DMO de pacientes pediátricos con infección vertical por VIH y su relación con factores de riesgo (TARGA, el estado inmunológico, la etapa de la enfermedad, las concentraciones plasmáticas de vitamina D en sangre y la ingesta de calcio).

\section{Materiales y Métodos}

\section{Sujetos de estudio}

Los participantes del estudio fueron 53 niños y adolescentes con infección por VIH adquirida por transmisión vertical, de ambos sexos, con edades comprendidas entre 8 y 18 años, en control en cinco hospitales pediátricos de Santiago de Chile. Los criterios de exclusión fueron: enfermedades que podrían afectar la masa ósea, tales 
como trastornos endocrinos, insuficiencia renal o hepática y uso de medicamentos como corticosteroides o anticonvulsivantes.

\section{Parámetros a evaluar}

A partir de los registros médicos se recopiló información sobre la edad, la etapa clínica de los pacientes según el Centro para el Control y Prevención de Enfermedades $(\mathrm{CDC})^{12}$, la duración de la enfermedad, inicio de TARGA, la carga viral (copias ARN/ml), concentración de linfocitos T CD4 (céls $/ \mathrm{mm}^{3}$ ) y su porcentaje, concentración plasmática de 25-hidroxivitamina $\mathrm{D}$, fosfatasa alcalina, calcio y fósforo. La deficiencia de vitamina $\mathrm{D}$ se definió como una concentración $<20 \mathrm{ng} / \mathrm{ml}$.

La antropometría se realizó el mismo día que la densitometría ósea (DEXA). El peso se midió con ropa interior en una balanza Secca digital (sensibilidad $10 \mathrm{~g}$ y capacidad: $200 \mathrm{~kg}$ ) y la talla en un estadiómetro (sensibilidad: $1 \mathrm{~mm}$ ). Se determinó el índice de masa corporal (IMC) para cada sujeto dividiendo el peso en kilogramos por la estatura al cuadrado $\left(\mathrm{kg} / \mathrm{m}^{2}\right)$.

Los niños fueron evaluados nutricionalmente por z-score de IMC de acuerdo con las tablas OMS/FAO ${ }^{13}$. Se consideró eutrófico un IMC entre $-0,9 \mathrm{y}+0,9 \mathrm{DE}$, sobrepeso entre $\geq+1$ y $<+1,9 \mathrm{DE}$, obeso $\geq+2 \mathrm{DE}$, riesgo desnutrir $\leq-1 \mathrm{y}>-1,9 \mathrm{DE}$ y desnutrición $\leq-2 \mathrm{DE}$. Talla baja se definió como $\mathrm{T} / \mathrm{E} \leq-2 \mathrm{DE}$.

\section{Densidad mineral ósea}

La DMO de cuerpo total se midió por densitómetro DEXA Lunar Prodigy. Todos los participantes se evaluaron según protocolos estándar en el mismo equipo calibrado cada dos días. Se examinó la columna lumbar (L1-L4) y el cuello femoral. El contenido mineral óseo (CMO) se informó en gramos, mientras que la DMO (excluyendo la cabeza) en $\mathrm{g} / \mathrm{cm}^{2}$ y puntajes z. Las puntuaciones z de DMO se basaron en National Health y Nutrition Examination Survey III $^{14}$ ajustado por edad y sexo. Se consideró normal una puntuación $\mathrm{z}>-1 \mathrm{DE}$.

\section{Encuesta nutricional}

Se realizó registro de ingesta por medio de recordatorio de $24 \mathrm{~h}$ repetido tres veces (dos días de semana y un día de fin de semana). Se obtuvieron así las cantidades diarias ingeridas (en gramos); posteriormente a eso, se cuantifican nutrientes, con el programa Food processor SQL. Se calculó el porcentaje de adecuación dietaria recomendado por la ingesta dietaria de referencia (RDI $)^{15}$ para cada uno de los nutrientes. El uso de multivitamínicos también fue registrado.

\section{Análisis estadístico}

Los datos se expresaron como promedio y desviación estándar o mediana y rangos según la distribución de las variables cuantitativas evaluada en forma gráfica con gráficos de densidad de Kernell y la prueba de ShapiroWilks. Para evaluar las diferencias entre grupos se usaron el test Mann-Whitney o el test t-Student según la distribución de la variable, y la prueba de $\chi^{2}$. Se estudió la correlación entre variables mediante la correlación de Pearson o Spearman según distribución.

Finalmente, se realizaron modelos de regresión lineal considerando como variable respuesta la DMO y la asociación principal con los tipos de terapia antiretroviral (TARV) (en años de tratamiento con el medicamento X). En una primera instancia se estudiaron aquellas variables que podrían explicar la DMO, mediante una estrategia backward obteniendo de esta forma un modelo basal con las variables cuyo valor $p$ fuera menor que 0,2 . Luego, se estimó la asociación bivariada entre la DMO y los diferentes tratamientos; y en última instancia se ajustó este modelo con las variables obtenidas en el modelo basal, considerándose estadísticamente significativo un $\mathrm{p}<0,05$. Para los análisis se utilizó el programa Stata SE 11.2.

\section{Aspectos éticos}

Previo al inicio del estudio se obtuvo el consentimiento informado por escrito de los padres o sus cuidadores. El estudio fue aprobado por el Comité de Ética del Servicio Metropolitano de Salud Norte.

\section{Resultados}

Se estudiaron 53 niños infectados verticalmente por el VIH, con mediana de edad 13,6 años (8-18,5) de quienes $54,7 \%$ eran mujeres. Del total, $32,1 \%$ se encontraban con sobrepeso u obesidad, sólo 11,3\% en riesgo a desnutrir y $18,9 \%$ tenía talla baja (Tabla 1 ). No se encontraron diferencias significativas entre hombres y mujeres al analizar las variables antropométricas (Tabla 1).

La mayoría de los pacientes estaba en etapas avanzadas de la enfermedad (89\% B y C) al momento del estudio, principalmente en el grupo de las mujeres $(93,1 \%$ etapas B y C). En general, los pacientes se encontraban con un buen estado inmunológico y virológico. La mayoría presentaba una ausencia o moderada presencia de inmunosupresión ( $\approx 90 \%$ del grupo) y una carga viral indetectable $(58 \%)$ (Tabla 1).

El tiempo de uso de TARGA fue de 9,3 \pm 4,6 años, sin diferencia entre sexos. El promedio de tiempo de uso fue mayor en el caso de los inhibidores nucleósidos de la transcriptasa reversa (INTR) y menor en el de los inhibidores de las proteasas (IP) (Tabla 1). El 85\% de los pacientes recibió INTR; los más comúnmente utilizados fueron lamivudina (83\%) y zidovudina (72\%). El fuma- 


\begin{tabular}{|c|c|c|c|c|}
\hline & Hombre $(n=24)$ & Mujer $(n=29)$ & Valor $p$ & Total $(n=53)$ \\
\hline Edad años, mediana (rango) & $13,6(8,3-18,4)$ & $12,9(8-18,5)$ & $0,83^{*}$ & $13,6(8-18,5)$ \\
\hline Estado nutricional, \% (n) & & & $0,52^{* *}$ & \\
\hline Sobrepeso u obeso & $41,6(10)$ & $24,13(7)$ & & $32,1(17)$ \\
\hline Normal & $45,8(11)$ & $65,5(19)$ & & $56,6(30)$ \\
\hline Bajo peso & $12,5(3)$ & $10,3 \quad(3)$ & & $11,3 \quad(6)$ \\
\hline z T/E promedio (DE) & $-1,2(1,2)$ & $-1,1(0,9)$ & $0,56^{*}$ & $-1,1 \quad(1)$ \\
\hline$z<-2 D E \%(n)$ & $16,7(4)$ & $20,7(6)$ & $0,71^{* *}$ & $18,9(10)$ \\
\hline Tiempo total TARV, años (DE) & $9,1(4,2)$ & $9,5(4,9)$ & 0,61 * & $9,3(4,6)$ \\
\hline \multicolumn{5}{|l|}{ TARV, años (DE) } \\
\hline NRTI & $7,9(4,2)$ & $9 \quad(4,7)$ & $0,33^{*}$ & $8,5(4,5)$ \\
\hline NNRTI & $4,9(3,5)$ & $6,1(4,5)$ & $0,32^{*}$ & $5,5(4,1)$ \\
\hline $\mathrm{PI}$ & $4,8(4,6)$ & $3,8(4,6)$ & $0,60 *$ & $4,1(4,6)$ \\
\hline Etapa CDC \% (n) & & & $0,08^{* *}$ & \\
\hline $\mathrm{N}$ & $4,2(1)$ & $3,5 \quad(1)$ & & $3,8 \quad(2)$ \\
\hline A & $12,5(3)$ & $3,5 \quad(1)$ & & $7,6 \quad(4)$ \\
\hline B & $41,7(10)$ & $75,9(22)$ & & $60,4(32)$ \\
\hline \multirow[t]{2}{*}{ C } & $41,7(10)$ & 17,2 & & $28,3(15)$ \\
\hline & Hombres $(n=22)$ & Mujeres $(n=26)$ & & Total $(n=48)$ \\
\hline Conteo $\mathrm{CD} 4$ cels $/ \mathrm{mm}^{3}$ (mediana y rango) & $578,5(156-1.335)$ & $779(76-1.378)$ & $0,03^{*}$ & $616(76-1.378)$ \\
\hline \multicolumn{5}{|l|}{ Porcentaje pacientes con CD4 } \\
\hline$<200$ & $9,1 \quad(2)$ & $3,8 \quad(1)$ & $0,24^{* *}$ & $6,3 \quad(3)$ \\
\hline $200-500$ & $27,3 \quad(6)$ & $11,5 \quad$ (3) & & $18,7 \quad(9)$ \\
\hline$>500$ & $63,6(14)$ & $84,6(22)$ & & $75 \quad(36)$ \\
\hline \multicolumn{5}{|l|}{ Porcentaje CD4 (\%) } \\
\hline $0-14 \%$ & $9,1 \quad(2)$ & 3,9 (1) & $0,02^{* *}$ & $6,3 \quad$ (3) \\
\hline $15 \%-24 \%$ & $31,8 \quad(7)$ & 3,9 (1) & & $16,7 \quad(8)$ \\
\hline$\geq 25 \%$ & $59,1(13)$ & $92,3(24)$ & & $77,1(37)$ \\
\hline Carga viral, n (\%) & & & $0,74^{* *}$ & \\
\hline Indetectable & $59,1(13)$ & $57,7(15)$ & & $58,3(28)$ \\
\hline $1-400$ & 18,2 & $11,5 \quad$ (3) & & $14,6 \quad(7)$ \\
\hline $401-5.000$ & $13,6 \quad$ (3) & $11,5 \quad$ (3) & & $12,5 \quad(6)$ \\
\hline$>5.000$ & $9,1 \quad(2)$ & 19,2 & & $14,6 \quad(7)$ \\
\hline
\end{tabular}

T/E, Talla/edad; TARV, terapia antiretroviral; PI, inhibidores de las proteasas; NRTI, nucleósidos inhibidores de transcriptasa reversa; NNRTI, no nucleósidos inhibidores de la transcriptasa reversa; CDC, Centros para el Control de Enfermedades; ${ }^{*}$ Test Mann-Whitney; ${ }^{* *}$ Test chicuadrado.

rato de disoproxilo de tenofovir (TDF) se usó sólo en $5,6 \%$ de los pacientes. Setenta y cuatro por ciento de los niños usó inhibidores no nucleósidos de la transcriptasa reversa (INNTR) siendo efavirenz el más frecuente (49\%) y luego nevirapina (32\%). Los IP se usaron en $53 \%$ de los pacientes, siendo la más frecuente combinación lopinavir/ ritonavir (LOP/rtv) (43,3\%).

En cuanto al consumo de macronutrientes, $46 \%$ de los pacientes cumplía más de $90 \%$ de las recomendaciones para calorías de la $\mathrm{RDI}^{15}$ y $100 \%$ cumplía los requerimientos de proteínas. La ingesta de calcio fue baja, sólo $11 \%$ de los individuos consumía más de 90\% de las RDI y nadie cumplía las recomendaciones de consumo de vitamina $\mathrm{D}$. Además, se observó que 33,3\% de los niños presentaban deficiencia en las concentraciones plasmáticas de vitamina D (menor a $20 \mathrm{ng} / \mathrm{ml}$ ) (Tabla 2).

Al analizar los resultados de la DMO no hubo diferencias entre sexos en cuanto al compromiso ni en la columna, cadera ni cuerpo total (Tabla 2). El porcentaje de pacientes con $\mathrm{DMO} \leq-2 \mathrm{SD}$ en columna, cadera y cuerpo entero fue de $11,5 \%, 5,8 \%$ y $3,8 \%$ respectivamente, siendo la columna la más comprometida (Tabla 2). 


\begin{tabular}{|c|c|c|c|c|}
\hline Variable & Hombres $(n=24)$ & Mujeres $(n=28)$ & Valor $\mathrm{p}$ & Total $(n=52)$ \\
\hline z DMO columna \% (n) & & & 0,26 ** & \\
\hline$>-1$ & $62,5(15)$ & $53,6(15)$ & & $57,7(30)$ \\
\hline$<-1$ a $-1,99 \mathrm{DE}$ & $20,8(5)$ & $39,9(11)$ & & $30,8(16)$ \\
\hline$<-2 \mathrm{DE}$ & $16,7(4)$ & $7,1(2)$ & & $11,5(6)$ \\
\hline z DMO cadera \% (n) & & & $0,71 * *$ & \\
\hline$>-1$ & $70,8(17)$ & $78,6(22)$ & & 75 (39) \\
\hline$<-1 \mathrm{DE}$ a $-1,99 \mathrm{DE}$ & $20,8(5)$ & $17,8(5)$ & & $19,2(10)$ \\
\hline$<-2 \mathrm{DE}$ & $8,3(2)$ & $3,6(1)$ & & $5,8(3)$ \\
\hline z DMO cuerpo total \% (n) & & & $0,90 * *$ & \\
\hline$>-1$ & $73,8(17)$ & $78,6(22)$ & & $75(39)$ \\
\hline$<-1 \mathrm{DE}$ a $-1,99 \mathrm{DE}$ & $21,7(5)$ & $17,2(5)$ & & $19,2(10)$ \\
\hline$<-2 \mathrm{DE}$ & $4,4(1)$ & $3,5(1)$ & & $3,8(2)$ \\
\hline \multicolumn{5}{|l|}{ Análisis bioquímicos promedio (DE) } \\
\hline Calcio plasmático mg/dl & $9,8(0,7)$ & $9,4(0,4)$ & $0,05^{*}$ & $9,6(0,6)$ \\
\hline Fósforo plasmático mg/dl & $4,6(0,9)$ & $4,5(0,6)$ & $0,84^{*}$ & $4,5(0,7)$ \\
\hline Fosfatasas alcalinas UI/I & $238,5(63,5)$ & $206,1(132,9)$ & $0,22 *$ & $222,3(103,4)$ \\
\hline Vitamina D (ug/ml) & $28,4(17,1)$ & $24,7(11,4)$ & $0,39 *$ & $26,4(14,2)$ \\
\hline \multicolumn{5}{|l|}{ Ingesta dietética } \\
\hline Consumo > $90 \%$ de RDI $\%$ (n) & Hombres $(n=19)$ & Mujeres $(n=27)$ & & Total $(n=46)$ \\
\hline Kilocalorías & $21,1(4)$ & $62,9(17)$ & $0,005^{* *}$ & $45,7(21)$ \\
\hline Proteínas & 100 & 100 & & 100 \\
\hline Calcio & $5,3(1)$ & $14,8(4)$ & $0,31 * *$ & $10,9(5)$ \\
\hline Vitamina D & 0 & 0 & & 0 \\
\hline
\end{tabular}

Al comparar a los pacientes según estado nutricional (riesgo a desnutrir versus normo o sobrepeso) no se observó diferencia significativa ni en DMO ni contenido mineral óseo en la columna ni la cadera. Cuando se comparó la TARV, se observó que los individuos que usaron IP presentaron una significativa menor DMO en cadera, fémur y cuerpo entero en comparación con el grupo que no los utilizó $(\mathrm{p}<0,01)$.

Se observó correlación entre DMO y variables como z IMC, z T/E, gravedad de la enfermedad, años de tratamiento, TARGA usada y calcio plasmático.

En la construcción del modelo basal se probaron todas las variables que pudieran estar asociadas al z DMO de cadera, columna y cuerpo total, quedando finalmente determinado por las variables, z T/E, z IMC, clasificación actual de la enfermedad, masa magra/talla, masa magra de extremidades/talla y calcio plasmático. En el análisis bivariado con los tratamientos usados se observó que los IP tenían una asociación negativa sobre DMO de columna, cadera y cuerpo entero y los INTR influían sólo en la DMO de la columna. Al introducir el modelo basal se observó que todos los tratamientos perdieron significancia excepto los IP (Tabla 3).

\section{Discusión}

El principal hallazgo de este estudio fue que los niños y adolescentes con infección vertical por VIH presentaron un compromiso de la DMO si se comparan con los datos obtenidos de sujetos sanos de la misma edad cronológica del NHANES III ${ }^{14}$ y que los IP, son el único TARV que mantiene una asociación con DMO, al ajustar por variables del modelo basal. Estos hallazgos ya han sido documentados en otros estudios en niños infectados a nivel mundial ${ }^{4,6,15,16,18}$. Sin embargo, es difícil comparar resultados entre estudios ya que hay diferencias en los densitómetros usados, poblaciones de referencia, gravedad de la enfermedad y uso de TARV.

Lamentablemente no tenemos tablas de referencia de DMO para niños sanos en nuestro país; el único estudio chileno fue el de Blanco y cols. ${ }^{19}$ que estudio la DMO en adolescentes de 16-17 años sanos donde $21 \%$ de los adolescentes presentó una DMO de cuerpo total bajo-1DE y las mujeres tenían mayor compromiso óseo.

Dos de los pacientes $(4,6 \%)$ habían presentado fracturas durante la evolución de la enfermedad, uno presentó fractura en una pierna y antebrazo en distinto período y 


\begin{tabular}{|c|c|c|c|c|c|c|}
\hline & \multicolumn{2}{|c|}{ z DMO cadera } & \multicolumn{2}{|c|}{ z DMO columna } & \multicolumn{2}{|c|}{ z DMO cuerpo total } \\
\hline & B & p-valor & B & $\mathrm{p}$-valor & B & p-valor \\
\hline \multicolumn{7}{|l|}{ Modelo bivariado } \\
\hline IP & $-0,17$ & $<0,01$ & $-0,10$ & $<0,001$ & $-0,13$ & $<0,01$ \\
\hline R2 & \multicolumn{2}{|c|}{0,38} & \multicolumn{2}{|c|}{0,21} & \multicolumn{2}{|c|}{0,33} \\
\hline NNRTI & 0,05 & 0,31 & 0,03 & 0,42 & 0,01 & 0,67 \\
\hline R2 & \multicolumn{2}{|c|}{0,02} & \multicolumn{2}{|c|}{0,01} & \multicolumn{2}{|c|}{0,004} \\
\hline NRTI & $-0,06$ & 0,14 & $-0,04$ & 0,19 & $-0,06$ & 0,08 \\
\hline R2 & \multicolumn{2}{|c|}{0,04} & \multicolumn{2}{|c|}{0,04} & \multicolumn{2}{|c|}{0,06} \\
\hline \multicolumn{7}{|l|}{ Modelo multivariado } \\
\hline $\mid \mathrm{P}+$ mod.basal ${ }^{*}$ & $-0,13$ & $<0,001$ & $-0,06$ & 0,04 & $-0,08$ & $<0,004$ \\
\hline R2 aj & \multicolumn{2}{|c|}{0,60} & \multicolumn{2}{|c|}{0,42} & \multicolumn{2}{|c|}{0,66} \\
\hline NNRTI + mod.basal* & 0,02 & 0,5 & 0,02 & 0,50 & $-0,01$ & 0,75 \\
\hline R2 aj & \multicolumn{2}{|c|}{0,39} & \multicolumn{2}{|c|}{0,39} & \multicolumn{2}{|c|}{0,59} \\
\hline NRTI+ mod . basal* & $-0,04$ & 0,29 & $-0,02$ & 0,54 & $-0,04$ & 0,17 \\
\hline R2aj & \multicolumn{2}{|c|}{0,43} & \multicolumn{2}{|c|}{0,37} & \multicolumn{2}{|c|}{0,61} \\
\hline $\begin{array}{l}\text { DMO, densitometría óse } \\
\text { de extremidades/talla + } \\
\text { de las proteasas; NRTI, n } \\
\text { el número de predictores }\end{array}$ & $\begin{array}{l}\text { E }+z \\
\text { núsculc } \\
\text { ptasa r } \\
\text { es con }\end{array}$ & $\begin{array}{l}\text { clasificaci } \\
\text { xtremidade } \\
\text {; NNRTI, nc } \\
2 \text { con estr }\end{array}$ & $\begin{array}{l}\text { ual de la } \\
\text { R cuadra } \\
\text { ósidos } \\
\text { backwar }\end{array}$ & $\begin{array}{l}\text { medad }+ \\
\text { oeficiente } \\
\text { anscriptas }\end{array}$ & $\begin{array}{l}\text { nagra/tal } \\
\text { erminaci } \\
\text { sa; R2 aj, }\end{array}$ & $\begin{array}{l}\text { masa magr } \\
\text { inhibidore } \\
\text { ustado par }\end{array}$ \\
\hline
\end{tabular}

otro paciente en una pierna. En el análisis actual ambos presentaron una DMO baja de columna, cadera (-2,5 -2,1 y $-1,2-1,4$, respectivamente) y el paciente con antecedentes de más de una fractura tenía además compromiso del cuerpo entero (DMO -2). Este último paciente cumpliría con los requisitos de osteoporosis dados por ISCD 2013 para diagnosticar esta enfermedad en niños ${ }^{20}$. En adultos existen bastantes antecedentes que demuestran el mayor riesgo de fracturas en pacientes con infección por $\mathrm{VIH}^{2,3}$; en niños, sin embargo, la información es menor. Siberry y cols. ${ }^{21}$, en un estudio multicéntrico, prospectivo, entre los años 2004 y 2007, no encontraron una mayor incidencia de fracturas en niños con infección por VIH en comparación con niños expuestos al VIH pero no infectados. Estos resultados fueron luego comparados con la incidencia de fracturas en un segundo estudio realizado entre los años 2008 y $2014^{22}$. En éste, los autores sí observaron un mayor riesgo de fracturas comparado con el primer estudio, incluso después de ajustar por edad, sugiriendo que el riesgo de fracturas podría estar incrementado también en los niños.

El compromiso óseo secundario a esta enfermedad y la TARV usada afectan toda la composición del hueso. Yin y cols. ${ }^{23}$, en adultos jóvenes infectados verticalmente o en la adolescencia temprana, encontraron que no sólo impide alcanzar la masa ósea máxima, sino que además adelgaza el hueso cortical y la microestructura ósea trabecular es anormal, lo que provoca una resistencia ósea reducida. Estudios en animales e in vitro han sugerido que estas alteraciones en la estructura son múltiples y podrían deberse a un aumento de la actividad de los osteoclastos por incremento del receptor del factor $\mathrm{NF} \mathrm{kB}$, aumento de la remodelación ósea asociado a la producción de IL $6^{24,25}$, disminución de la diferenciación a osteoblastos desde la célula stem cell mesenquimática, junto a una disminución de los factores de transcripción de los osteoblastos y de IGF-1 producido por la interferencia de proteínas específicas del virus ${ }^{25}$.

Al evaluar los factores asociados a una menor DMO, se observó una asociación negativa con el zT/E, tiempo y gravedad de la enfermedad. Este hallazgo ya ha sido descrito en diversas poblaciones ${ }^{4,25}$ y se debería al efecto de la enfermedad y TARV sobre el metabolismo óseo por mecanismos antes descritos, lo que irían progresando en el tiempo.

La carga viral y el estado inmunológico actual no se asociaron a los cambios de DMO. Estos índices cambian rápidamente dependiendo del tratamiento, mientras que los cambios en el hueso son más lentos y requieren meses para ser detectados ${ }^{26}$.

Los años con TARGA muestran una asociación negativa con la DMO. En adultos la iniciación del TARV está asociada con una pérdida de 2 a $6 \%$ de la DMO en las primeras 96 semanas de tratamiento ${ }^{27}$ y tienen dos a 
cuatro veces más riesgo de osteoporosis que los pacientes expuestos al VIH, pero no infectados ${ }^{28}$. En este estudio, si bien los años de TARV tienen un efecto deletéreo sobre la DMO, son los IP los que causan mayor repercusión, concordando con otros estudios en población infantil $1^{4,6,16,17}$. En la población estudiada, $53 \%$ de los pacientes recibió un IP dentro de su tratamiento, siendo LOP/rtv el más frecuentemente usado. Ritonavir altera la expresión génica, la cual induciría una actividad inflamatoria local con la consecuente reducción en la actividad de los osteoblastos ${ }^{18}$ y la exposición a la asociación de LOP/rtv produce además una disminución en la actividad de las fosfatasas alcalinas de los osteoblatos, así como una disminución en los depósitos de calcio y un aumento en la actividad osteoclástica ${ }^{2}$. Por otro lado, $12 \%$ de los pacientes que recibieron LOP/ rtv recibieron además stavudina y $10 \%$ tenofovir, los que se consideran moduladores de la disminución de la masa ósea ${ }^{2}$ lo que aumentaría más el riesgo.

No se observó correlación entre las concentraciones plasmáticas de vitamina D y la DMO, probablemente porque fue sólo una medida en el tiempo y ésta no da información sobre las concentraciones plasmáticas promedio que han tenido los pacientes durante su enfermedad. Es relevante que $33 \%$ de estos niños y adolescentes presentan concentraciones plasmáticas de vitamina $\mathrm{D}$ inferiores a $20 \mathrm{ng} / \mathrm{dl}$. Estudios en distintas poblaciones con infección por VIH han reportado altas tasas de deficiencia de esta vitamina, que van de 23 a $92 \%{ }^{1,2,29,30}$. Pérez y cols. ${ }^{31}$, en población de hombres chilenos infectados, encontraron concentraciones plasmáticas bajas de esta vitamina en 94\% de los pacientes. Ellos plantean que la alta deficiencia en estos pacientes podría deberse a una alteración de $1 \alpha$-hidroxilación que disminuye la producción y acción del metabolito activo de la vitamina $\mathrm{D}$, a pesar de concentraciones plasmáticas de vitamina $\mathrm{D}$ normales y a que algunos $\mathrm{ARV}$, tales como IP y efavirenz, suprimen la actividad de 25 y $1 \alpha$-hidroxilasa o activan la destrucción de 25-OHD y 1,25-hidroxivitamina D3 ${ }^{32}$. Este es un hallazgo que debe considerarse ya que la deficiencia de vitamina D no sólo se ha asociado a una menor DMO en estos pacientes, sino que también se ha asociado a una progresión de la enfermedad, falla del TARV y muerte ${ }^{33}$.

Asociado a lo anterior, si bien no se encontró asociación entre el consumo de calcio y vitamina D con DMO, Overtone y cols. ${ }^{34}$, demostraron que la suplementación de calcio y vitamina $\mathrm{D}$ en el primer año después de iniciado la TARV minimizó la pérdida de DMO. En nuestro estudio, sólo $11 \%$ de los niños cumplen con más de $90 \%$ de las recomendaciones de consumo de calcio según edad y nadie cubre los requerimientos de vitamina D. Esto no se diferencia de lo que ocurre en el país, donde, salvo los menores de 4 años, más de $90 \%$ de los sujetos de todas las edades, no alcanzan a ingerir los requerimientos de estos nutrientes ${ }^{35}$.
Este estudio tiene limitaciones. Primero, es un estudio transversal y, por lo tanto, incapaz de evaluar el cambio de la masa ósea durante el período de tratamiento. Por consiguiente, se hace necesario un estudio longitudinal prospectivo para ver los cambios en el tiempo. Segundo, muchos adolescentes han recibido varios esquemas de TARV durante su enfermedad, por lo que nuestra capacidad para evaluar el impacto de los medicamentos específicos fue limitada, si bien pudimos determinar que hubo una asociación entre las distintas familias de ARV y DMO. En tercer lugar, hay variables importantes que afectan el compromiso óseo que no se midieron en este estudio, como el desarrollo puberal y cuantía de ejercicio. Durante la adolescencia, las hormonas sexuales interfieren en la antropometría y composición corporal y aumentan directamente la masa ósea. La infección por VIH/SIDA es una enfermedad crónica en que el retraso en el desarrollo puberal puede ocurrir con frecuencia, por lo que la edad cronológica puede ser mayor que la edad biológica y se hace necesario no sólo comparar por edad a los pacientes sino además, por estadio puberal ${ }^{36,37}$. Por otro lado, la actividad física es un factor ambiental importante que influye positivamente en la acumulación de masa ósea, principalmente durante la pubertad ${ }^{38}$. Otro de los puntos a considerar dentro de estas limitaciones es el hecho que $18 \%$ de nuestra población presentaba talla baja y se sabe que el DEXA no es el mejor método para evaluar DMO en estos pacientes ya que determina la cantidad de hueso dividida por el área de estudio; de esta manera, también se toma en cuenta el espesor y tamaño del hueso, lo que llevaría a que pacientes con talla baja tengan valores de densitometría baja siendo realmente normal. En estos pacientes estaría indicada la densitometría volumétrica, ya que, a diferencia del método anterior, determina la masa ósea que está contenida en un determinado quantum de volumen de hueso y no se ve influenciada por el tamaño del hueso ${ }^{39}$. Sin embargo, esta técnica está poco disponible en Chile, su costo es mayor y produce mayor irradiación que el DEXA tradicional ${ }^{39}$.

En conclusión, este estudio muestra que los niños y adolescentes con infección por VIH tendrían una DMO más baja por edad en comparación con los datos de NHANES III. La gravedad de la enfermedad, el compromiso de la talla, z IMC, los años de TARV, principalmente el uso de IP, están relacionados con este problema. Estudios han demostrado que estos pacientes, en la edad adulta, tienen más riesgo de osteoporosis y fracturas por lo que en la niñez es necesario tratar de obtener la máxima masa ósea. Para esto, es necesario constituir un equipo multidisciplinario de profesionales (infectólogo, pediatra y/o nutriólogo, nutricionista, endocrinólogo, enfermera, asistente social, etc) que realicen controles frecuentes según edad (lactantes mensualmente y en pacientes mayores, según evolución), que aseguren un estado 
nutricional adecuado a lo largo de toda la enfermedad, un control estricto de las consecuencias de TARV, estimular la realización de mayor actividad física (deportes, horas de juego al aire libre, evitar el uso de pantallas) y la ingesta adecuada de calcio y vitamina $\mathrm{D}$. En este último punto, recomendamos que estos pacientes se controlen con concentraciones plasmáticas de 25 hidroxi-vitamina $\mathrm{D}$ al menos una vez al año para identificar y tratar a pacientes con deficiencia. Está pendiente evaluar cuál es el rango más adecuado de vitamina $\mathrm{D}$ para estos pacientes y si es necesaria la suplementación.

\section{Resumen}

Los cambios en la densidad mineral ósea (DMO) son comunes en adultos infectados con virus de la inmunodeficiencia humana (VIH). Existen pocos estudios que evalúen el compromiso óseo en niños. Objetivo: Evaluar la DMO en niños infectados verticalmente por VIH.
Métodos: Se estudiaron 53 niños infectados (8-18) de cinco hospitales. Se registró severidad de enfermedad, evaluación nutricional, vitamina D (25-OHD) y estado inmunológico. La DMO se midió mediante densitometría. Se utilizó análisis descriptivo, comparación de medias y regresión lineal simple y múltiple. Resultados: $\mathrm{El}$ $88,7 \%$ estaban en estadio B y C, $57 \%$ eran eutróficos y $18,9 \%$ tenían talla baja. El $33,3 \%$ presentaba niveles de $25-\mathrm{OHD}<20 \mathrm{ng} / \mathrm{ml}$. El 11\%, 6\% y $4 \%$ de los niños tenían DMO $<2 \mathrm{DE}$ en cadera, columna y cuerpo entero, respectivamente. La DMO se correlacionó con IMC, talla, severidad de enfermedad y años de tratamiento. Sólo inhibidores de las proteasas (IP) mantuvieron su significancia al ajustar por otras variables. Conclusión: Los niños infectados con VIH tuvieron DMO más baja por edad comparados con datos de NHANES III. La severidad de la enfermedad, talla, zIMC, los años de tratamiento con anti-retrovirales, principalmente IP, están relacionados con la reducción de la masa ósea.

\section{Referencias bibliográficas}

1.- Sudjaritruk T, Bunupuradah T, Aurpibul L, Kosalaraksa P, Kurniati N, Prasitsuebsai W, et al. Bone-D Study Group. Adverse bone health and abnormal bone turnover among perinatally HIV-infected Asian adolescents with virological suppression. HIV Med. 2017; 18: 235-44. https://doi.org/10.1111/hiv.12418.

2.- Broder S. The development of antiretroviral therapy and its impact on the HIV-1/AIDS pandemic. Antiviral Res. 2010; 85: 1-18. doi: 10.1016/j.antiviral.2009.10.002.

3.- Paccou J, Viget N, Legrout-Gerot I, Yazdanpanah Y, Cortet B. Bone loss in patients with HIV infection. Joint Bone Spine. 2009; 76: 637-41. doi: 10.1016/j.jbspin.2009.10.003.

4.- Jacobson D L, Spiegelman D, Duggan C, Weinberg G A, Bechard L, Furuta L, et al. Predictors of bone mineral density in human immunodeficiency virus-1 infected children. J Pediatr Gastroenterol Nutr. 2005; 41: 339-46. PMID: 16131991.

5.- O’Brien K O, Razavi M, Henderson R A, Caballero B, Ellis K J. Bone mineral content in girls perinatally infected with HIV. Am J Clin Nutr 2001; 73: 821-6. DOI: 10.1093/ ajen/73.4.821

6.- Puthanakit T, Saksawad R, Bunupuradah T, Wittawatmongkol O, Chuanjaroen T, Ubolyam $\mathrm{S}$, et al. Prevalence and risk factors of low bone mineral density among perinatally HIV-infected Thai adolescents receiving antiretroviral therapy. Acquir Immune Defic Syndr. 2012; 61: 477-83. doi: [10.1097/ QAI.0b013e31826ea89b].

7.- Brown T T, Qaqish R B. Antiretroviral therapy and the prevalence of osteopenia and osteoporosis: a meta-analytic review. AIDS 2006; 20: 2165-74. DOI: 10.1097 QAD.0b013e32801022eb.

8.- Mora S, Sala N, Bricalli D, Zuin G, Chiumello $\mathrm{G}$, Viganò A. Bone mineral loss through increased bone turnover in HIV-infected children treated with highly active antiretroviral therapy. AIDS 2001; 15: 1823-9. PMID: 11579244.

9.- Miller T L, Easley K A, Zhang W, Orav E J, Bier D M, Luder E, et al. Maternal and infant factors associated with failure to thrive in children with vertically transmitted human immunodeficiency virus-1 infection: The prospective, $\mathrm{P} 2 \mathrm{C} 2$ human immunodeficiency virus multicenter study. Pediatrics 2001; 108 : 1287-96. PMCID: PMC4383837.

10.- Mahoney E M, Donfield S M, Campbell H, Kaufman F, Gertner J M. HIV-associated immune dysfunction and delayed pubertal development in a cohort of young hemophiliacs. J Acquir Immune Defic Syndr 1999; 21: 333-37. PMID: 10428113.

11.- Gilsanz V, Nelson D A. Primer on the metabolic bone disorders and disorders of mineral metabolism. Favus, M.J. 5th ed. Washington: American Society for Bone and Mineral Research 2003; 72-7.

12.- CDC: 1994 Revised Classification system for human immunodeficiency virus infection in children less than 13 years of age. MMWR Morbid Mortal Wkly Reo1994; 43 (RR-12): 1-10. https://www.cdc.gov/mmwr/PDF/rr/ rr4312.pdf.

13.- WHO Multicentre Growth Reference Study Group. WHO Child Growth Standards: Length/ height-for-age, weight-for-age, weight-forlength, weight-for-height and body mass indexfor-age: Methods and development. Geneva: World Health Organization, 2006. https://www. who.int/childgrowth/standards/technical_report/ en/

14.- Looker A C, Borrud L G, Hughes J P, Fan B, Shepherd J A, Melton L J 3rd. Lumbar spine and proximal femur bone mineral density, bone mineral content, and bone area: United States, 2005-2008. Vital Health Stat 11. 2012; 251: 1-132. PMID: 24261130.

15.- Recommended Dietary Allowances: 10th Edition. The National Academies Press, Washington, DC. Retrieved 2011. 03-30.

16.- DiMeglio L A, Wang J, Siberry G K, Miller T L, Geffner M E, Hazra R, et al. Pediatric HIVAIDS Cohort Study (PHACS). Bone mineral density in children and adolescents with perinatal HIV infection. AIDS. 2013; 27 : 211-20. doi: 10.1097/QAD.0b013e32835a9b80.

17.- Lima L R, Silva R C, Giuliano Ide C, Sakuno T, Brincas S M, Carvalho A P. Bone mass in children and adolescents infected with human immunodeficiency virus J Pediatr. 2013; 89: 91-9. doi: 10.1016/j.jped.2013.02.014.

18.- Malizia A P, Vioreanu M H, Doran P P, Powderly W G. HIV1 protease inhibitors selectively induce inflammatory chemokine expression in primary human osteoblasts. Antiviral Res 2007; 74: 72-6. DOI: 10.1016/j. antiviral.2006.12.003.

19.- Blanco E, Reyes M, Burrows R A, Martínez S $\mathrm{M}$, Gahagan S. Low bone mineral density in a cohort of normal, overweight and obese Chilean adolescents. J Clin Nurs 2011; 20: 3577-9. doi: 10.1111/j.1365-2702.2011.03959.x. 
20.- Gordon C M, Leonard M B, Zemel B S, International Society for Clinical Densitometry. 2013 Pediatric Position Development Conference: executive summary and reflections. J Clin Densitom 2014; 17: 219-4. doi: 10.1016/j.jocd.2014.01.007.

21.- Siberry G K, Li H, Jacobson D. Fracture risk by HIV infection status in perinatally HIV-exposed children. AIDS Res Hum Retroviruses. 2012; 28: 247-50. doi: [10.1089/ aid.2011.0064].

22.- Mirani G, Williams P L, Chernoff M, Abzug M J, Levin M J, Seage G R, et al. Changing trends in complications and mortality rates among US youth and young adults with HIV infection in the era of combination antiretroviral therapy. Clin Infect Dis 2015; 61: 1850-61. doi: 10.1093/cid/civ687.

23.- Yin M T, Lund E, Shah J, Zhang C A, Foca M, Neu N, et al. Lower peak bone mass and abnormal trabecular and cortical microarchitecture in young men infected with HIV early in life. AIDS. 2014; 28: 345-53. doi: 10.1097/QAD.0000000000000070.

24.- Vikulina T, Fan X, Yamaguchi M, Roser-Page S, Zayzafoon M, Guidot D M, et al. Alterations in the immuno-skeletal interface drive bone destruction in HIV-1 transgenic rats. Proc Natl Acad Sci USA 2010; 107: 13848-53. doi: 10.1073/pnas.

25.- Stagi S, Bindi G, Galluzzi F, Galli L, Salti $\mathrm{R}$, de Martino M. Changed bone status in human immunodeficiency virus type 1(HIV1) perinatally infected children is related to low serum free IGF-I. Clin Endocrinol 2004; 61: 692-9. DOI: $10.1111 /$ j.13652265.2004.02150.x.

26.- Gordon C M, Bachrach L K, Carpenter T
O, Crabtree N, El-Hajj Fuleihan G, Kutilek $\mathrm{S}$, et al. Dual energy X-ray absorptiometry interpretation and reporting in children and adolescents: the 2007 ISCD Pediatric Official Positions. J Clin Densitom 2008; 11: 43-58. doi: 10.1016/j.jocd.2007.12.005.

27.- Sax P E, Wohl D, Yin M T, Post F, DeJesus E, Saag M, et al. Tenofovir alafenamide versus tenofovir disoproxil fumarate, coformulated with elvitegravir, cobicistat, and emtricitabine, for initial treatment of HIV-1 infection: two randomised, double- blind, phase 3 , noninferiority trials. Lancet $2015 ; 385$ : $2606-$ 15. doi: 10.1016/S0140-6736(15)60616-X.

28.- Zuccotti G, Viganò A, Gabiano C, Giacomet V, Mignone F, Stucchi S, et al. Antiretroviral therapy and bone mineral measurements in HIV-infected youths. Bone 2010; 46: 1633-8. doi: 10.1016/j.bone.2010.02.029.

29.- Huh S Y, Gordon C M. Vitamin D deficiency in children and adolescents: epidemiology, impact and treatment. Rev Endocr Metab Disord 2008; 9: 161-70. doi: 10.1007/s11154-007-9072-y.

30.- Rutstein R, Downes A, Zemel B, Schall J, Stallings V. Vitamin D status in children and young adults with perinatally acquired HIV infection. Clin Nutr 2011; 30: 624-8. doi: 10.1016/j.clnu.2011.02.005.

31.- Pérez C, Ceballos M E. High frequency of hypovitaminosis $\mathrm{D}$ and low bone mineral density in a sample of HIV-infected men in Chile. Rev Chilena Infectol 2014; 3: 44-6. DOI: $10.4067 / \mathrm{S} 0716-10182014000100006$.

32.- Madeddu G, Spanu A, Solinas P, Calia GM, Lovigu C, Chessa F, et al. Bone mass loss and vitamin D metabolism impairment in HIV patients receiving highly active antiretroviral therapy. Q J Nucl Med Mol Imaging 2004; 48 :
39-48. PMID: 15195003.

33.- Mansueto P, Seidita A, Vitale G, Gangemi $\mathrm{S}$, Iaria C, Cascio A. Vitamin D deficiency in HIV infection: not only a bone disorder. Biomed Res Int 2015; 2015: 735615. doi: 10.1155/2015/735615.

34.- Overton E T, Chan E S, Brown T T, Tebas P, McComsey G A, Melbourne K M, et al. High-dose vitamin $\mathrm{D}$ and calcium attenuate bone loss with antiretroviral therapy initiation: a randomized trial. Ann Intern Med 2015; 162 : 815-24. doi: [10.7326/M14-1409].

35.- Encuesta nacional de consumo alimentario. http://web.minsal.cl/sites/default/files/ENCA_ FINAL_DIC_2014.

36.- Eckard A R, Mora S. Bone health in HIVinfected children and adolescents. Curr Opin HIV AIDS 2016; 11: 294-300. doi: 10.1097/ COH.0000000000000270.

37.- Jacobson D L, Lindsey J C, Gordon C M, Moye J, Hardin D S, Mulligan K, Aldrovandi G M; Pediatric AIDS Clinical Trials Group P1045 team. Total body and spinal bone mineral density across Tanner stage in perinatally HIV-infected and uninfected children and youth in PACTG 1045. AIDS 2010; 24: 687-96. doi: [10.1097/QAD.0b013e328336095d].

38.- MacKelvie K J, Petit M A, Khan K M, Beck T J, McKay H A. Bone mass and structure are enhanced following a 2-year randomised controlled trial of exercise in prepubertal boys. Bone 2004; 34: 755-64. DOI: 10.1016/j. bone.2003.12.017

39.- Nelson D A, Koo W W K. Interpretation of absorptiometry bone mass measurements in the growing skeleton: issues and limitations. Calcif Tissue Int 1999; 65: 1-3. https://eurekamag. com/pdf/010/010869756.pdf. 